\title{
The Fourier Transform in Optics: Analogous Experiment and Digital Calculus
}

\author{
Petre Cătălin Logofătu${ }^{1}$, Victor Nascov ${ }^{2}$ and Dan Apostol ${ }^{1}$ \\ ${ }^{1}$ National Institute for Laser, Plasma and Radiation Physics \\ ¿Universitatea Transilvania Braşov \\ Romania
}

\section{Introduction}

Discrete optics and digital optics are fast becoming a classical chapter in optics and physics in general, despite their relative recent arrival on the scientific scene. In fact their spectacular blooming began precisely at the time of the computer revolution which made possible fast discrete numerical computation. Discrete mathematics in general and discrete optics in particular although predated digital optics, even by centuries, received a new impetus from the development of digital optics. Formalisms were designed to deal with the specific problems of discrete numerical calculation. Of course, these theoretical efforts were done not only for the benefit of optics but of all quantitative sciences. Diffractive optics in general and the newly formed scientific branch of digital holography, turned out to be especially suited to benefit from the development of discrete mathematics. One reason is that the optical diffraction in itself is a mathematical transform. An ordinary optical element such as the lens turned out to be a genuine natural optic computer, namely one that calculates the Fourier transform (Goodman, 1996, chapter 5).

The problem is that the discrete mathematics is not at all the same thing as continuous mathematics. For instance for the most common theoretical tool in diffractive optics, the continuous (physical) Fourier transform (CFT) we have a discrete correspondent named discrete Fourier transform (DFT). We need DFT because the Fourier transform rarely yields closed form expressions and generally can be computed only numerically, not symbolically. Of course, no matter how accurate, by its very nature DFT can be only an approximation of CFT. But there is another advantage offered by DFT which inclines the balance in favour of discrete optics. The reason is somehow accidental and requires some explanation. It is the discovery of the Fast Fourier transform, for short FFT, (Cooley \& Tukey, 1965), which was followed by a true revolution in the field of discrete optics because of the reduction with orders of magnitude of the computation time, especially for large loads of input data. FFT stirred also an avalanche of fast computation algorithms based on it. The property that allowed the creation of these fast algorithms is that, as it turns out, most diffraction formulae contain at their core one or more Fourier transforms which may be rapidly calculated using the FFT. The method of discovering a new fast algorithm is oftentimes to reformulate the diffraction formulae so that to identify and isolate the Fourier transforms it contains. We contributed ourselves to the development of the field with the creation of an 
improved algorithm for the fast computation of the discrete Rayleigh-Sommerfeld transform and a new concept of convolution: the scaled linearized discrete convolution (Nascov \& Logofătu, 2009). The conclusion is that we want to use DFT, even if CFT would be a viable alternative, because of its amazing improvement of computation speed, which makes feasible diffraction calculations which otherwise would be only conjectures to speculate about.

Here is the moment to state most definitely the generic connection between the digital holography and the DFT, more specifically the FFT, as was outlined in the pioneer work of (Lohmann \& Paris, 1967), and since then by the work of countless researchers, of which, for lack of space and because it is not our intent to write a monography about the parallel evolution of digital holography and DFT, we will mention only a few essential works that deal both subjects in connection to each other. There is, of course, a vast deal of good textbooks and tutorials dedicated to the fundamentals of the Fourier transform, continuous or discrete (Arfken \& Weber, 2001; Bracewell, 1965; Brigham, 1973; Bringdahl \& Wyrowsky, 1990; Collier et al., 1971; Goodman, 1996; Lee, 1978; Press et al., 2002 and Yaroslavsky \& Eden, 1996), but in our opinion a severe shortcoming of the textbooks listed above is the fact that, in our opinion none offers a complete and satisfactory connection between the two formalisms, such as the expression of DFT in terms of CFT. We use DFT in place of CFT but we do not know exactly what is the connection between them! In Yaroslavsky \& Eden, 1996, chapter 4 and Collier, 1971, chapter 9, a correspondence is worked out between DFT and CFT, namely that the value of the DFT is equal to the value of the CFT at the sample points in the Fourier space, but this is valid only for band-limited functions and it is not rigorous. (Strictly speaking DFT can be applied only to band-limited functions, but this is an unacceptable restriction; many of the functions of interest are not band-limited. In general we have to approximate and to compensate for the assumed approximations.) Generally those textbooks fail to link in the proper manner the fertile but inapplicable in practice in itself field of discrete optics, to continuous, physical optics, where the experiments take place and we can take advantage of the progress of the discrete optics. In our own scientific research activity in the field of digital optics we encountered the difficulty almost at every step [Apostol \& al., 2007 (a); Apostol et al., 2007 (b); Logofătu et al., 2009 and Logofătu et al., 2010]. In two previous occasions (Logofătu \& Apostol, 2007 and Nascov et al., 2010) we attempted to express the physical meaning of DFT, to put it in the terms of CFT using the Fourier series as an intermediary concept. Together with the present work this continued effort on our part will hopefully prove useful to all those who undertake projects in discrete optics and they are hampered by the gap between DFT and CFT, discrete mathematics, digital computers on the one hand and real physical experiments on the other hand.

With the above rich justification we did not exhaust by far the uses of DFT and the need to rigorously connect it to $\mathrm{CFT}$. Apart from all virtual computation, which also requires the connection between DFT and CFT to be worked out, digital holography present special hybrid cases where a discrete and a continuous character are both assumed. For instance the recording of holograms can be made using Charged Coupled Devices (CCD) which are discrete yet they work in the real continuous physical world. The same is valid for the other end of holography, the reconstruction or the playback of the holograms. For this purpose today are used devices such as Spatial Light Modulators (SLM) of which one can also say they are hybrid in nature, digital and analogous in the same time. For such devices as CCDs and SLMs one has to switch back and forth between CFT and DFT and think sometimes in terms of one of the formalisms and some other times in terms of the other. Here we should 
mention the pioneer work of Lohmann and Paris for the compensation of the "digital" effect, so to speak, in their experiments with digital holograms (Lohmann \& Paris, 1967).

For physical reasons in optics, when dealing with images, a 2D coordinate system with the origin in the center of the image is used. However, FFT deals with positive coordinates only, which means they are restricted to the upper right quadrant of the 2D coordinate system. In order to work in such conditions one has to perform a coordinate conversion of the input image before the calculations, and also the final result of the calculations has to be converted in order to have the correct output image. The conversion involves permutations of quadrants and sign for the values of the field. The positive coordinates are necessary for the application of the FFT algorithm, which makes worthwhile the complication by its very fast computation time. It is possible to work only with positive coordinates because the discrete Fourier transform assumes the input and the output being infinite and periodic (Logofătu \& Apostol, 2007). The disadvantage of this approach is its counterintuitive and artificial manner. The correspondence to the physical reality is not simple and obvious. In our practically-oriented paper we used a natural, physical coordinate system, (hence negative coordinates too), and we performed a coordinate conversion of the images only immediately before and after the application of the FFT. In this way, the correspondence to the physical reality is simple and obvious at all times and this gives to our approach a more intuitive character. Precisely for this reason in chapter 4 we present an alternative method for converting the physical input so that can be used by the mathematical algorithm of FFT and for converting the mathematical output of FFT into data with physical meaning, a method that do not use permutation of submatrices, which may be preferable for large matrices, a method based on the shifting property of the Fourier transform applied to DFT.

In order to keep the mathematics to a minimum the equations were written as for the $1 \mathrm{D}$ case whenever possible. The generalization to $2 \mathrm{D}$ is straightforward and the reader should keep in mind at all times the generalization to the 2D case, the real, physical case. The equations are valid for the 1D case too, of course, but the 1D case is just a theoretical, imaginary case.

\section{The translation of DFT in the terms of CFT or the top-down approach}

\subsection{Short overview of the current situation}

In our efforts to bridge the two independent formalisms of CFT and DFT first we used more of a top-down approach, working from the principles down to specific results (Logofătu \& Apostol, 2007). In mathematics there are three types of Fourier transforms: (I) CFT, (II) the Fourier series and (III) DFT. Only the first type has full physical meaning, and can be accomplished for instance in optics by Fresnel diffraction using a lens or by Fraunhofer diffraction. The third type is a pure mathematical concept, although it is much more used in computation than the previous two for practical reasons. These three types of Fourier transforms are independent formalisms, they can stand alone without reference to one another and they are often treated as such, regardless of how logic and necessity generated one from another. Because only CFT has physical meaning, the other two types of transforms are mathematical constructs that have meaning only by expressing them in terms of the first. In order to be able to do this we have to present the three transforms in a unifying view. To our knowledge no mathematics or physics textbooks present such a unifying view of the three types of Fourier transforms, although all the necessary knowledge lies in pieces in the literature. An integrated unifying presentation of the three 
types of Fourier transforms has then the character of a creative review, so to speak. In this paper such a unifying view is presented. The Fourier transform of type II, the Fourier series, besides its own independent worth, is shown to be an intermediary link between the Fourier transforms of types I and III, a step in the transition between them. Also some concrete cases are analyzed to illustrate how the discrete representation of the Fourier transform should be interpreted in terms of the physical Fourier transform and how one can make DFT a good approximation of CFT. In the remainder of this chapter such a unifying view is presented. The Fourier transform of type II, the Fourier series, besides its own independent worth, is shown to be an intermediary link between the Fourier transforms of types I and III, a step in the transition between them. Also some concrete cases are analyzed to illustrate how the discrete representation of the Fourier transform should be interpreted in terms of the physical Fourier transform and how one can make DFT a good approximation of CFT.

\subsection{Fourier transforms}

Suppose we have a function $g(t)$ and we are interested in its Fourier transform function $G(f)$. Here $t$ is an arbitrary variable (may be time or a spatial dimension) and $f$ is the corresponding variable from the Fourier space (like time and frequency or space and spatial frequencies). We work in the $1 \mathrm{D}$ case for convenience but the extrapolation to $2 \mathrm{D}$, (i.e. the optic case, the one we are interested), is straightforward.

The three types of Fourier transforms are defined as following: (I) continuous, i.e. the calculation of the transform $G(f)$ is done for functions $g(t)$ defined over the real continuum, that is the interval $(-\infty \ldots+\infty)$, and the transformation is made by integration over the same interval

$$
\mathrm{G}(\mathrm{f})=F\{\mathrm{~g}\}(\mathrm{f})=\int_{-\infty}^{+\infty} \mathrm{g}(\mathrm{t}) \exp (-\mathrm{i} 2 \pi \mathrm{ft}) \mathrm{dt}
$$

$F$ being the operator for Fourier transform, with $G$ being also generally defined over the real continuum, (II) Fourier series, where the function to be transformed is defined over a limited range $(0 \ldots \Delta t)$ of the continuum, and instead of a Fourier transformed function defined over $(-\infty \ldots+\infty)$ we have a series which represents the discrete coefficients of the Fourier expansion

$$
G_{n}=\frac{1}{\Delta t} \int_{-\Delta t / 2}^{\Delta t / 2} g(t) \exp (-i 2 \pi n t / \Delta t) d t, n=-\infty, \ldots, \infty
$$

and (III) the purely discrete Fourier transform where a list of numbers is transformed into another list of numbers by a summation procedure and not by integration

$$
G_{q}=\sum_{p=-N / 2}^{N / 2-1} g_{p} \exp (-i 2 \pi q p / N), q=-N / 2, \ldots, N / 2-1
$$

(For clarity purposes, in this chapter we use consistently $m$ and $n$ to indicate the periodicity, and $p$ and $q$ to indicate the sampling of $g$ and $G$ respectively.) Although these three types of Fourier transforms can be considered independent formalisms, they are strongly connected. Indeed, the second may be considered a particularization of the first, by restricting the class 
of input functions $g$ to periodic functions only and then to take into consideration for calculation purposes only one period from the interval $(-\infty \ldots+\infty)$ over which $g$ is defined. The third can be considered a particularization of the second, by requesting not only that the functions $g$ to be periodic, but also discrete, to have values only at even spaced intervals $\delta t$. Therefore the third type of Fourier transform may be considered an even more drastic particularization of the most general Fourier transform I.

That is the top-down approach. It is possible another approach, a bottom-up one, in which the second type of Fourier transform is considered a generalization of the third type, or a construct made starting from the third type, and the same thing can be said about the relation between the first and the second type. In order to pass from the Fourier transform type III to type II, in the list $g_{m}$ which is a discrete sampling made at equal intervals $\delta t$ we make $\delta t \rightarrow 0$ and $N \rightarrow \infty$, which results in the list $g_{m}$ becoming a function of continuous argument and the numbers $G_{n}$ are not anymore obtained by summation as in Eq. (3) but by integration as in Eq. (2). Now we are dealing with the Fourier transform type II. Making the function $g$ periodic, by imposing

$$
\mathrm{g}(\mathrm{t}+\mathrm{m} \Delta \mathrm{t})=\mathrm{g}(\mathrm{t})
$$

where $m$ is an arbitrary integer, and making the period $\Delta t \rightarrow \infty$, the discrete coefficients $G_{n}$ become a continuum and we are back to the Fourier transform of type I. But we will deal with this approach in more detail in chapter 3.

\subsection{From continuous Fourier transform to Fourier series}

Since the formalism of the Fourier transform of type I is the most general and the only one with full physical meaning, we will express the two other formalisms in its terms. As we said before, if the input function $g$ is periodic, then the corresponding output function $G$ degenerates into a series. Indeed, if $g$ has the period $\Delta t$ as in Eq. (4) then the corresponding $G$ can be written as

$$
\begin{aligned}
\mathrm{G}(\mathrm{f}) & =\sum_{\mathrm{m}=-\infty}^{+\infty} \int_{\mathrm{m}-1 / 2) \Delta \mathrm{t}}^{(\mathrm{m}+1 / 2) \Delta \mathrm{t}} \mathrm{g}(\mathrm{t}) \exp (-\mathrm{i} 2 \pi \mathrm{ft}) \mathrm{dt}=\sum_{\mathrm{m}=-\infty}^{+\infty} \int_{-\Delta t / 2}^{\Delta \mathrm{t} / 2} \mathrm{~g}(\mathrm{t}) \exp [-\mathrm{i} 2 \pi \mathrm{f}(\mathrm{t}+\mathrm{m} \Delta \mathrm{t})] \mathrm{dt}= \\
& =\int_{-\Delta \mathrm{t} / 2}^{\Delta \mathrm{t} / 2} \mathrm{~g}(\mathrm{t}) \exp (-\mathrm{i} 2 \pi \mathrm{ft}) \mathrm{dt} \sum_{\mathrm{m}=-\infty}^{+\infty} \exp (-\mathrm{i} 2 \pi \mathrm{mf} \Delta \mathrm{t})
\end{aligned}
$$

Without rigorous demonstration we will state here that the infinite sum of exponentials in the uttermost right hand side of Eq. (5) is an infinite sum of delta functions called the comb function,

$$
\sum_{m=-\infty}^{+\infty} \exp (-\mathrm{i} 2 \pi \mathrm{mf} \Delta \mathrm{t})=\frac{1}{\Delta t} \sum_{n=-\infty}^{\infty} \delta\left(\mathrm{f}-\frac{\mathrm{n}}{\Delta \mathrm{t}}\right) \equiv \operatorname{comb}(\Delta \mathrm{t} f)
$$

Indeed, one may check that the sum of exponentials is 0 everywhere except for $f$ of the form $n / \Delta t$ when it becomes infinite. When $f$ is of the form $n / \Delta t$ all the members of the infinite sum are equal to 1 . When $f$ differs however slightly from $n / \Delta t$, the phaser with which we can represent the exponentials in the uttermost left hand side of Eq. (6) in the complex plane runs with incremental equidistant strokes from 0 to $2 \pi$ when the index $m$ of the sum grows 
incrementally with the result that the contributions of the terms to the sum cancels each other out, although not necessarily term by term. (One should not confuse the delta function $\delta(t)$ with the sampling interval $\delta t$.) Introducing (6) into (5) one obtains

$$
G(f)=\frac{1}{\Delta t} \sum_{n=-\infty}^{\infty} \delta\left(f-\frac{n}{\Delta t}\right) \int_{-\Delta t / 2}^{\Delta t / 2} g(t) \exp (-i 2 \pi n t / \Delta t) d t=\sum_{n=-\infty}^{\infty} G_{n} \delta\left(f-\frac{n}{\Delta t}\right)
$$

where $G_{n}$ were defined in Eq. (2). One can see from Eq. (7) that the Fourier series are a particular case of CFT, namely the Fourier transform $G$ of a periodic function $g$ of period $\Delta t$ is a sum of delta functions of arguments shifted with $1 / \Delta t$ intervals and with coefficients $G_{n}$ that are the same as the coefficients defined in Eq. (2). Actually it is the coefficients from Eq. (2) that are the Fourier series, and not the function defined in Eq. (7), but the correspondence is obvious.

In optical experiments one may see a good physical approximation of the Fourier series when a double periodic mask, with perpendicular directions of periodicity, modulates a plane monochromatic light wave and the resulting optical field distribution is Fourier transformed with the help of a lens. In the back focal plane of the lens, where the Fourier spectrum is formed, we have a distribution of intensely luminous points along the directions of periodicity. The luminous points do not have, of course, a rigorous delta distribution, they are not infinitely intense and they have non-zero areas. This departure from ideal is due to the fact that neither the mask nor the plane wave are ideal. The image that is Fourier transformed is neither infinite nor rigorously periodic, since the intensity of the plane wave decreases from a maximum in the centre to zero towards the periphery. But such an experiment is a good physical illustration of the mathematics involved in Eq. (7)

The difference between CFT and the Fourier series can be seen also from the inverse perspective of the Fourier expansion of $g$,

$$
\begin{aligned}
g(t) & =F^{-1}\{G\}(t)=\int_{-\infty}^{+\infty} G(f) \exp (i 2 \pi f t) d t= \\
& =\int_{-\infty}^{+\infty} \sum_{n=-\infty}^{\infty} G_{n} \delta\left(f-\frac{n}{\Delta t}\right) \exp (i 2 \pi f t) d t=\sum_{n=-\infty}^{\infty} G_{n} \exp (i 2 \pi n t / \Delta t)
\end{aligned}
$$

The difference is that in the case of a periodic function the Fourier expansion is a sum and not an integral anymore.

\subsection{The discrete Fourier transform}

For computation purposes we cannot use always a function $g$ defined over the continuum but a sampled version instead. There are many reasons that make the sampling of $g$ necessary. It is possible that the function $g$, representing a physical signal, an optical field for instance, is not known a priori and only a detected sample of it can be known. It is possible that the function $g$ cannot be integrated because it is too complicated or it cannot be expressed in closed form functions or it causes numerical instabilities in the calculation of CFT. A very important reason might be the fact that the function $g$ may be the result of calculations too as in the case of computer-generated Fourier holograms. In this case, $g$ is known only as a 2D matrix of numbers. (For simplicity, however, we will continue to work 
with 1D functions as long as possible.) Then we have to sample the input function, and we can do that with the help of the comb function; this type of sampling will yield the value of $g$ at evenly spaced intervals of chosen value $\delta t$ and it can be written as

$$
\mathrm{g}^{\mathrm{s}}(\mathrm{t})=\mathrm{g}(\mathrm{t}) \delta \mathrm{t} \sum_{\mathrm{p}=-\infty}^{\infty} \delta(\mathrm{t}-\mathrm{p} \delta \mathrm{t})=\delta \mathrm{t} \sum_{\mathrm{m}=-\infty}^{\infty} \mathrm{g}(\mathrm{p} \delta \mathrm{t}) \delta(\mathrm{t}-\mathrm{p} \delta \mathrm{t})=\delta \mathrm{t} \sum_{\mathrm{p}=-\infty}^{\infty} \mathrm{g}_{\mathrm{p}} \delta(\mathrm{t}-\mathrm{p} \delta \mathrm{t})
$$

where $g_{p}$ are the sampled values $g(p \delta t)$ and $p$ is an arbitrary integer. The superscript "s" stands for "sample". One may notice that when $\delta t \rightarrow 0$ we have $g^{s} \rightarrow g$.

Now in order to obtain $G$ we may apply CFT to $g$, but we may prefer to apply instead DFT defined in Eq. (3) for the reasons already mentioned in section 1. A sampled Fourier spectrum does not mean necessarily that the Fourier transform has to be performed discretely; we may perform it continuously and then sample the resulting continuous spectrum. But this would be a waste of effort. It is preferable to compute the Fourier spectrum discretely. But what physical correspondence has the discrete transform in reality, where the transform is continuous? To find out one needs to express DFT in terms of CFT. In other words using CFT of the sampled function we try to obtain the Fourier spectrum also under a sampled form. Here we can make use of the Fourier series, which now prove to be, as we stated before, an intermediary link between CFT and DFT.

We know from subsection 2.2 that the CFT of a periodic function is a discrete even spaced function. Since we want $G$ to be discrete, then we have to make $g$ periodic. The input function $g$ is not generally periodic but in most practical cases it has values only over a finite domain of arguments. Suppose $g$ is non-zero only for arguments in the interval $t \in(-\Delta t / 2$ $\ldots \Delta t / 2)$. We define a periodic function $g^{p}$ as

$$
\mathrm{g}^{\mathrm{p}}(\mathrm{t})=\sum_{\mathrm{m}=-\infty}^{\infty} \mathrm{g}(\mathrm{t}+\mathrm{m} \Delta \mathrm{t})
$$

The superscript " $\mathrm{p}$ " means that $g^{p}$ is the periodic version of $g$. A function that is non-zero only in the interval $(-\Delta t / 2 \ldots \Delta t / 2)$, does not have to be sampled to infinity, but only where has non-zero values. Sampling $g$ over the interval $(-\Delta t / 2 \ldots \Delta t / 2)$ gives us the same quantity of information as sampling $g^{p}$ to infinity if, for simplicity, we choose the sampling interval $\delta t$ so that

$$
\Delta \mathrm{t}=\mathrm{N} \delta \mathrm{t}
$$

where $N$ is an integer. To make the input function both discrete and periodic, we have to combine the forms (9) and (10) of $g$ together, and, taking into account (11), we obtain

$$
\mathrm{g}^{\mathrm{sp}}(\mathrm{t})=\delta \mathrm{t} \sum_{\mathrm{m}=-\infty}^{\infty} \sum_{\mathrm{p}=-\mathrm{N} / 2}^{\mathrm{N} / 2-1} \mathrm{~g}_{\mathrm{p}} \delta(\mathrm{t}+\mathrm{m} \Delta \mathrm{t}-\mathrm{p} \delta \mathrm{t})
$$

Here the superscript "sp" means that the function $g s p$ is both sampled (discrete) and periodic. We know from the general properties of CFT of its reciprocal character (Bracewell, 1965; Goodman, 1996) and that the inverse CFT is very similar to CFT itself [see Eqs. (1) and (8); DFT has a similar property]. A double CFT reproduces the input function up to an inversion of coordinates. Therefore, if the CFT of a periodic function is an even-spaced 
discrete sampling, then the CFT of an even-spaced discrete sampling has to be a periodic function. Let us check this assertion by calculating the CFT of (12)

$$
\begin{aligned}
\mathrm{G}^{\mathrm{sp}}(\mathrm{f}) & =\delta \mathrm{t} \int_{-\infty}^{\infty} \sum_{\mathrm{m}=-\infty}^{\infty} \sum_{\mathrm{p}=-\mathrm{N} / 2}^{\mathrm{N} / 2-1} \mathrm{~g}_{\mathrm{p}} \delta(\mathrm{t}+\mathrm{m} \Delta \mathrm{t}-\mathrm{p} \delta \mathrm{t}) \exp (-\mathrm{i} 2 \pi \mathrm{ft}) \mathrm{dt}= \\
& =\delta \mathrm{t} \sum_{\mathrm{p}=-\mathrm{N} / 2}^{\mathrm{N} / 2-1} \mathrm{~g}_{\mathrm{p}} \exp (-\mathrm{i} 2 \pi \mathrm{f} \mathrm{p} \delta \mathrm{t}) \sum_{\mathrm{m}=-\infty}^{\infty} \exp (\mathrm{i} 2 \pi \mathrm{fm} \Delta \mathrm{t})
\end{aligned}
$$

We make use again of the property (6) and obtain

$$
\begin{aligned}
\mathrm{G}^{\mathrm{sp}}(\mathrm{f}) & =\delta \mathrm{t} \sum_{\mathrm{p}=-\mathrm{N} / 2}^{\mathrm{N} / 2-1} \mathrm{~g}_{\mathrm{p}} \exp (-\mathrm{i} 2 \pi \mathrm{f} \mathrm{p} \delta \mathrm{t}) \frac{1}{\Delta \mathrm{t}} \sum_{\mathrm{q}=-\infty}^{\infty} \delta\left(\mathrm{f}-\frac{\mathrm{q}}{\Delta \mathrm{t}}\right)= \\
& =\frac{1}{\mathrm{~N}} \sum_{\mathrm{q}=-\infty}^{\infty} \delta\left(\mathrm{f}-\frac{\mathrm{q}}{\Delta \mathrm{t}}\right)_{\mathrm{p}=-\mathrm{N} / 2}^{\mathrm{N} / 2-1} \mathrm{~g}_{\mathrm{p}} \exp (-\mathrm{i} 2 \pi \mathrm{qp} / \mathrm{N})
\end{aligned}
$$

We notice that $G^{s p}$ is periodic with the period $\Delta f=1 / \delta t$, because adding $n / \delta t$ to the argument $f$ this causes only a reindexation with $n N$ of the infinite sum of delta functions that does not cause any modification to $G^{s p}$ precisely because the sum is infinite. We also notice that the coefficients of the delta functions are, up to a multiplication constant, the DFT of Eq. (3). We may rewrite then $G^{s p}$ as

$$
G^{s p}(f)=\frac{1}{N} \sum_{n=-\infty}^{\infty} \sum_{q=-N / 2}^{N / 2-1} G_{q} \delta\left(f-\frac{q}{\Delta t}-\frac{n}{\delta t}\right)
$$

In the expression (15) the periodic character of $G s p$, namely of period $1 / \delta t$, is more clearly visible than in Eq. (14). Also $G^{s p}$ is sampled at intervals of $\delta f=1 / \Delta t$. Both $g^{s p}$ and $G^{s p}$ are discrete and periodic. This is connected with the property of the function comb that it is invariant to CFT (Bracewell, 1965; Goodman, 1996), in other words the CFT of the comb function is also the comb function. The sampling of $g$ is made with a comb function and it was to be expected to retrieve the comb function in the expression of $G^{s p}$. One may say that the DFT of a sampled function $g$ is the CFT of the comb function weighted with $g^{p}$ and the result is a comb function weighted with $G^{p}$. It should be noted that $G^{s p}$ has the same number of distinct elements as $g s p, N$. This is to be expected since through Fourier transform no information is lost but only represented differently. We should be able to retrieve the same amount of information from $G$ as from $g$, therefore the number of samples should be the same for both functions. It is also noteworthy the inverted correspondence between the sampling intervals and the periods of $g^{s p}$ and Gsp. The sampling interval of Gsp is the inverse of the period of $g^{s p}$, and the period of $G^{s p}$ is the inverse of the sampling interval of $g^{s p}$.

One may see now that the correspondence between CFT and DFT given in references (Collier et al., 1971; Yaroslavsky \& Eden, 1996) is of a different kind than that shown above. In those references the output function $G$ is not discrete. Only the sampled values of $G$ correspond to the DFT of only the sampled values of $g$. Also our relation between CFT and DFT has the advantage of illustrating better some properties of DFT such as its cyclic or toroidal character (Collier et al., 1971). 
Eqs. $(12,15)$ represent the physical equivalent of DFT. Only for a function like $g^{s p}$, periodic and consisting of evenly spaced samples, can one calculate the Fourier transform as in Eq. (3), i.e. discretely, and such functions do not exist in reality, and one may wonder what is the usefulness of it all. There is usefulness inasmuch as $g^{s p}$ relates to $g$ and $G^{s p}$ to $G$, and they are related because we built $g^{s p}$ starting from $g$. But constructing $g^{s p}$ we adapted $g$ for the purpose of discrete computing and we departed from the original $g$ and consequently from G. Now we have to find how close are the CFT and the physical equivalent of DFT performed on $g$ and how we can bring them closer. But before that we think we should try the reciprocal approach, the bottom-up approach as one can call it, starting from the simplest formalism, the DFT and arriving at CFT, of course, again via the Fourier series, which seem to be the accomplished intermediary.

\section{The translation of CFT in the terms of DFT or the bottom-up approach}

The Fourier (or harmonic) analysis is a methodology used to represent a periodic function into a series of harmonic functions. The harmonic functions are well known elementary functions. Fourier analysis is applicable only for linear systems, where the principle of linear superposition is valid.

Let $g(t)$ be a real or complex periodic function, having the period $\Delta t$. The set of functions

$$
\Psi_{k}(t)=\exp \left(i 2 \pi f_{k} t\right), f_{k}=\frac{k}{\Delta t}, k=-\infty, \ldots,+\infty
$$

are the harmonic functions of $g$. Except for the constant function $\psi_{0}(t)=1$, all the other functions in the set exhibit oscillations with quantized frequencies $f_{k}$, which are integer multiples of $f_{1}=1 / \Delta t$, called the fundamental frequency. These functions repeat periodically over the whole real axis. The real and imaginary parts of $g$ are identical, but they are phase shifted: the real part has a phase delay of $\pi / 2$ (a quarter of a period) relative to the imaginary part. This infinite set of harmonic functions is an orthonormal set over the range of $t \in[-\Delta t / 2, \Delta t / 2]$ :

$$
\left\langle\Psi_{\mathrm{m}}, \Psi_{\mathrm{n}}\right\rangle \stackrel{\text { def }}{=} \frac{1}{\Delta \mathrm{t}} \cdot \int_{-\Delta \mathrm{t} / 2}^{\Delta \mathrm{t} / 2} \Psi_{\mathrm{m}}(\mathrm{t}) \Psi_{\mathrm{n}}^{*}(\mathrm{t}) \mathrm{dt}=\frac{1}{\Delta \mathrm{t}} \int_{-\Delta \mathrm{t} / 2}^{\Delta \mathrm{t} / 2} \exp \left[\mathrm{i} 2 \pi\left(\mathrm{f}_{\mathrm{m}}-\mathrm{f}_{\mathrm{n}}\right) \mathrm{t}\right] \mathrm{dt}=\delta_{\mathrm{mn}}= \begin{cases}0, & \mathrm{~m} \neq \mathrm{n} \\ 1, & \mathrm{~m}=\mathrm{n}\end{cases}
$$

The Sturm-Liouville theorem proves that a function $f$ respecting the Dirichlet conditions can be expressed as a linear combination of the harmonic functions (Arfken \& Weber, 2001, chapter 9).

$$
\mathrm{g}(\mathrm{t})=\sum_{\mathrm{k}=-\infty}^{\infty} \mathrm{c}_{\mathrm{k}} \Psi_{\mathrm{k}}(\mathrm{t}), \quad \mathrm{c}_{\mathrm{k}}=\left\langle\mathrm{g}, \Psi_{\mathrm{k}}\right\rangle=\frac{1}{\Delta \mathrm{t}} \int_{-\Delta \mathrm{t} / 2}^{\Delta \mathrm{t} / 2} \mathrm{~g}(\mathrm{t}) \Psi_{\mathrm{k}}^{*}(\mathrm{t}) \mathrm{dx}=\frac{1}{\Delta \mathrm{t}} \int_{-\Delta \mathrm{t} / 2}^{\Delta \mathrm{t} / 2} \mathrm{~g}(\mathrm{t}) \exp \left(-\mathrm{i} 2 \pi \mathrm{f}_{\mathrm{k}} \mathrm{t}\right) \mathrm{dt}
$$

This expansion is called Fourier series and the coefficients $c_{k}$ are called Fourier coefficients. Theoretically, there is an infinite number of Fourier coefficients. However, above a certain cut-off order, their amplitudes become very small and we can neglect them. The abscissa of the spectrum is proportional to the frequency. The frequencies corresponding to the spikes are multiples of the fundamental spatial frequency $1 / \Delta t$. At the same time, the multiples order is the index of the coefficient. For example, if we notice a strong spectral component at 
the $10^{\text {th }}$ position, we say that the $10^{\text {th }}$ harmonic, of frequency $f_{10}=10 f_{1}$, is one of the dominant harmonics of the spectrum. Since only a few number of spectral harmonics have significant amplitudes, we say that the given function $g$ can be well approximated by a superposition of a few Fourier harmonics.

The bidimensional (2D) Fourier series extends the regular Fourier series to two dimensions and is used for harmonic analysis of periodic functions of two variables. If $\Delta x$ and $\Delta y$ are the periods of the $g(x, y)$ function along the directions defined by the $x$ and $y$ variables, we define two fundamental angular frequencies: $f_{x}=1 / \Delta x$ and $f_{y}=1 / \Delta y$. The basis of $2 \mathrm{D}$ Fourier series expansion is built up from 2D Fourier harmonics, which are products of two simple 1D harmonics:

$$
\Psi_{\mathrm{mn}}(\mathrm{x}, \mathrm{y})=\exp \left(\mathrm{i} 2 \pi \mathrm{mf}_{\mathrm{x}} \mathrm{x}\right) \exp \left(\mathrm{i} 2 \pi \mathrm{nf}_{\mathrm{y}} \mathrm{y}\right), \quad \mathrm{m}, \mathrm{n}=0, \pm 1, \pm 2, \ldots, \pm \infty
$$

The Fourier series of the function $g(x, y)$ is double indexed, and the Fourier coefficients form a matrix.

$$
\begin{aligned}
& \mathrm{g}(\mathrm{x}, \mathrm{y})=\sum_{\mathrm{m}=-\infty}^{\infty} \sum_{\mathrm{n}=-\infty}^{\infty} \mathrm{c}_{\mathrm{mn}} \Psi_{\mathrm{mn}}(\mathrm{x}, \mathrm{y}) \\
& \mathrm{c}_{\mathrm{mn}}=\left\langle\mathrm{g}, \Psi_{\mathrm{mn}}\right\rangle=\frac{1}{\Delta \mathrm{x} \Delta \mathrm{y}} \int_{-\Delta \mathrm{x} / 2}^{\Delta \mathrm{x} / 2} \mathrm{~d} \mathrm{e} \exp \left(-\mathrm{i} 2 \pi \mathrm{mf}_{\mathrm{x}} \mathrm{x}\right) \int_{-\Delta \mathrm{y} / 2}^{\Delta \mathrm{y} / 2} \mathrm{dyg}(\mathrm{x}, \mathrm{y}) \exp \left(-\mathrm{i} 2 \pi \mathrm{nf}_{\mathrm{y}} \mathrm{y}\right)
\end{aligned}
$$

The continuous Fourier transform (CFT) may be understood by analyzing how the spectrum of the periodic function $g$ changes as a result of enlarging its period or the gradual change of the spectrum. The larger the period $\Delta t$ of $g$, the smaller the fundamental frequency $\delta \mathrm{f}=2 \Pi / \Delta t$ is, and the quantized set of angular frequencies $f_{k}=k \delta f$ are bunching together. When defining the function $g$ for an infinite period, reproducing its characteristic pattern only once, without reproducing it periodically, while outside we set it to equal zero, the function $g$ is no more periodic, or we can say that we have extended its period to infinity, $\Delta t \rightarrow \infty$. In this limit case the spectrum is no more discrete, but it becomes continuous. Related to the continuous spectrum, we mention some facts:

a. The difference between two consecutive quantized frequencies turns infinitesimal: $f_{k+1}-f_{k}=1 / \Delta t=\delta f \rightarrow 0$, so we replaced the discrete values $f_{k}$ by a continuous quantity $f$.

b. All the Fourier coefficient amplitudes shrank to zero. For this reason we replaced the Fourier coefficients by the quantities $\Delta t c_{k}$, which do not shrank to zero but remain finite and they became the new instruments of practical interest for describing the function $g$.

$$
\Delta t c_{k}=\int_{-\Delta t / 2}^{\Delta t / 2} g(t) \Psi_{k}^{*}(t) d t=\int_{-\Delta t / 2}^{\Delta t / 2} g(t) \exp \left(-i 2 \pi f_{k} t\right) d t, \quad \lim _{\Delta t \rightarrow \infty} \Delta t c_{k}=\int_{-\infty}^{\infty} g(t) \exp \left(-i 2 \pi f_{k} t\right) d t
$$

c. The integer index $k$ turns into a continuous variable when $\Delta t \rightarrow 0$, hence it is more appropriate to denote the Fourier coefficients replacements $\Delta t c_{k}$ by a continuous function $G(f)$, that we call Fourier transform of the $g$ function:

$$
G(f)=\lim _{\Delta t \rightarrow \infty} \Delta t c_{k}=\int_{-\infty}^{\infty} g(t) \exp (-i 2 \pi f t) d t
$$


d. The Fourier series from Eq. (18) approximates an integral, and on the limit $\Delta t \rightarrow \infty$ the series converge to that integral:

$$
g(t)=\lim _{\Delta t \rightarrow \infty} \sum_{k=-\infty}^{\infty} c_{k} \Psi_{k}(t)=\int_{-\infty}^{\infty} G(f) \exp (i 2 \pi f t) d f
$$

The rationale shown above for the transition from Fourier series to CFT is similar to the one shown elsewhere (Logofătu \& Apostol, 2007). Therefore, if the function $g$ is not periodic, it cannot be decomposed into a series of Fourier harmonics, but into a continuous superposition of Fourier harmonics, called Fourier integral. The Fourier integral decomposition is possible providing that the modulus of the non periodic function $g$ can be integrated over the whole real axis, that is the integral $\int_{-\infty}^{\infty}|g(t)| d t$ should exist (and be finite). Very common types of functions that fulfil this condition, largely used in practical applications are the functions with finite values over a compact interval and with zero values outside that interval. We implicitly assumed that the function $g$ considered above is of that type.

Now let us consider the definition of CFT (22) and the relation used to decompose the nonperiodic function $g$ into the Fourier integral (23). We notice that each transform is the inverse of each other:

$$
\mathrm{G}(\mathrm{f})=\int_{-\infty}^{\infty} \mathrm{g}(\mathrm{t}) \exp (-\mathrm{i} 2 \pi \mathrm{t}) \mathrm{dt}, \quad \mathrm{g}(\mathrm{t})=\int_{-\infty}^{\infty} \mathrm{G}(\mathrm{f}) \exp (\mathrm{i} 2 \pi \mathrm{ft}) \mathrm{dt}, \quad \mathrm{g}(\mathrm{t}) \stackrel{\text { Fourier }}{\longleftrightarrow} \mathrm{G}(\mathrm{f})
$$

We say the functions $g$ and $G$ form a pair of Fourier transforms. The function $G$ is obtained by applying the direct Fourier transform to the function $g$, while the function $g$ is obtained by applying the inverse Fourier transform to the function $G$.

The bidimensional (2D) Fourier transform extends the Fourier transform to two dimensions and is used for two variables functions, which should satisfy a similar condition: the integral $\int_{-\infty}^{\infty} \int_{-\infty}^{\infty}|g(x, y)| d x d y$ should exist and be finite. The Fourier integral is double:

$$
\begin{aligned}
& G\left(f_{x}, f_{y}\right)=\int_{-\infty}^{\infty} \int_{-\infty}^{\infty} g(x, y) \exp \left[-i 2 \pi\left(f_{x} x+f_{y} y\right)\right] d x d y, \\
& g(x, y)=\int_{-\infty}^{\infty} \int_{-\infty}^{\infty} G\left(f_{x}, f_{y}\right) \exp \left[i 2 \pi\left(f_{x} x+f_{y} y\right)\right] d f_{x} d f_{y}
\end{aligned}
$$

While the 1D Fourier transform can be used as an illustration, or as an approximation of the 2D Fourier transform, in the special cases where the input function $g$ does not depend on one or two coordinates, but three or more, although mathematically treatable, they present no interest for the physicist, because the 3D limitation of the world restricts practical interest to maximum 2D Fourier transform.

DFT has the purpose to approximate the CFT, and it is used for reasons of computation speed convenience. Although DFT is an independent formalism in itself, it was formulated so that it converges to the genuine CFT. DFT needs the function $g(t)$ as a set of a finite number $N$ of samples, taken at $N$ equidistant sample points, within a $\Delta t$ length interval: 


$$
\mathrm{t}_{\mathrm{m}}=\mathrm{m} \Delta \mathrm{t} / \mathrm{N}, \quad \mathrm{g}_{\mathrm{m}}=\mathrm{g}\left(\mathrm{t}_{\mathrm{m}}\right), \quad \mathrm{m}=-\mathrm{N} / 2,-\mathrm{N} / 2+1, \ldots, \mathrm{N} / 2-1
$$

In practical applications the function $g$ is only given as a set of samples, and even if one knows its analytical expression, in most cases it's not possible to determine its Fourier transform by analytical calculus.

The definition of DFT can be established after a series of approximations. First, one approximates the Fourier transform by a Fourier series, which is defined as a set of coefficients associated to a set of equidistant frequencies. For this purpose we extend the domain of the sampled function to the whole real axis, making the function periodic, with the period of $\Delta t$ which contains the entire initial definition domain of the function, in order to be able to expand it in Fourier series. The harmonic functions used as a decomposition basis are sampled functions too:

$$
\Psi_{\mathrm{nm}}=\Psi_{\mathrm{n}}\left(\mathrm{t}_{\mathrm{m}}\right)=\exp \left(\mathrm{i} 2 \pi \mathrm{f}_{\mathrm{n}} \mathrm{t}_{\mathrm{m}}\right)=\exp (\mathrm{i} 2 \pi \mathrm{mn} / \mathrm{N}), \quad \mathrm{m}, \mathrm{n} \in \mathrm{Z}
$$

We modify the definition of the scalar product of these functions replacing the integral by a sum that approximates it:

$$
\begin{aligned}
& \frac{1}{\Delta \mathrm{t}} \int_{-\Delta \mathrm{t} / 2}^{\Delta \mathrm{t} / 2} \Psi_{\mathrm{m}}(\mathrm{t}) \Psi_{\mathrm{n}}^{*}(\mathrm{t}) \mathrm{dt} \approx \frac{1}{\Delta \mathrm{t}} \sum_{\mathrm{k}=-\mathrm{N} / 2}^{\mathrm{N} / 2-1} \Psi_{\mathrm{m}}\left(\mathrm{t}_{\mathrm{k}}\right) \Psi_{\mathrm{n}}^{*}\left(\mathrm{t}_{\mathrm{k}}\right) \frac{\Delta \mathrm{t}}{\mathrm{N}}=\frac{1}{\mathrm{~N}} \sum_{\mathrm{k}=-\mathrm{N} / 2}^{\mathrm{N} / 2-1} \Psi_{\mathrm{mk}} \Psi_{\mathrm{nk}}^{*} \text {, } \\
& \left\langle\Psi_{\mathrm{m}}, \Psi_{\mathrm{n}}\right\rangle \stackrel{\operatorname{def}}{=} \frac{1}{\mathrm{~N}_{\mathrm{k}=-\mathrm{N} / 2}} \sum_{\mathrm{mk}}^{\mathrm{N} / 2-1} \Psi_{\mathrm{nk}}^{*}=\frac{1}{\mathrm{~N}_{\mathrm{k}=-\mathrm{N} / 2}} \sum_{\mathrm{N} / 2-1} \exp \left(\mathrm{i} 2 \pi \mathrm{k} \frac{\mathrm{m}-\mathrm{n}}{\mathrm{N}}\right)=\delta_{\mathrm{mn}}^{(\mathrm{N})}=\left\{\begin{array}{ll}
0, & \mathrm{~m}-\mathrm{n} \neq \mathrm{pN}, \\
1, & \mathrm{~m}-\mathrm{n}=\mathrm{pN},
\end{array}, \mathrm{p} \in \mathbf{Z}\right.
\end{aligned}
$$

There are only $N$ distinct discrete harmonic functions, which are linear independent and can build up an orthonormal basis, because they repeat periodically: $\psi_{n \pm N}(t)=\psi_{n}(t)$. The Fourier coefficients will be calculated in the same way, approximating the integral by a sum:

$$
c_{n}=\frac{1}{\Delta t} \int_{-\Delta t / 2}^{\Delta t / 2} g(t) \Psi_{n}^{*}(t) d t \approx \frac{1}{\Delta t} \sum_{k=-N / 2}^{N / 2-1} g\left(t_{k}\right) \Psi_{n}^{*}\left(t_{k}\right) \frac{\Delta t}{N}=\frac{1}{N} \sum_{k=-N / 2}^{N / 2-1} g_{k} \exp \left(-i 2 \pi \frac{n k}{N}\right)
$$

There are only a limited set of $N$ Fourier coefficients, because they reproduce themselves with the $N$ period too, $c_{n \pm N}=c_{n}$. The original discrete function $g$ can be expanded into a series of $N$ discrete harmonic functions:

$$
g_{m}=g\left(t_{m}\right)=\sum_{k=-N / 2}^{N / 2-1} c_{k} \Psi_{k}\left(t_{m}\right)=\sum_{k=-N / 2}^{N / 2-1} c_{k} \Psi_{k m}=\sum_{k=-N / 2}^{N / 2-1} c_{k} \exp \left(i 2 \pi \frac{k m}{N}\right)
$$

At this point we can define the discrete Fourier transform: it is a sampled function $G$ whose samples are the set of $N$ Fourier coefficients approximately calculated by sums in Eq. (29): $G_{n}=N c_{n}, n=-N / 2,-N / 2+1, \ldots, N / 2-1$. The samples of $G$ are obtained applying a transform to the samples of $g$ and they can be inverted in order to yield back the samples of $g$ from that of $\mathrm{G}$ as shown below in Eq. (31).

$$
\begin{aligned}
& \mathrm{G}_{\mathrm{n}}=\sum_{\mathrm{m}=-\mathrm{N} / 2}^{\mathrm{N} / 2-1} \mathrm{~g}_{\mathrm{m}} \exp \left(-\mathrm{i} 2 \pi \frac{\mathrm{mn}}{\mathrm{N}}\right), \quad \mathrm{g}_{\mathrm{m}}=\frac{1}{\mathrm{~N}_{\mathrm{n}=-\mathrm{N} / 2}} \mathrm{G}_{\mathrm{n}}^{\mathrm{N} / 2-1} \exp \left(\mathrm{i} 2 \pi \frac{\mathrm{mn}}{\mathrm{N}}\right), \\
& \mathrm{m}, \mathrm{n}=-\mathrm{N} / 2,-\mathrm{N} / 2+1, \ldots, \mathrm{N} / 2-1 \mathrm{~g} \stackrel{\mathrm{DFT}}{\longrightarrow} \mathrm{G}
\end{aligned}
$$


The two sets of samples from $g$ and $G$ form a pair of discrete Fourier transforms. The transform is a linear one and can be expressed by means of a square matrix of $N \times N$ dimensions:

$$
\overrightarrow{\mathrm{G}}=\hat{\mathrm{W}} \cdot \overrightarrow{\mathrm{g}}, \quad \overrightarrow{\mathrm{g}}=\hat{\mathrm{W}}^{-1} \cdot \overrightarrow{\mathrm{G}}, \quad \hat{\mathrm{W}}_{\mathrm{mn}}=\mathrm{w}_{\mathrm{N}}^{\mathrm{mn}}, \quad \hat{\mathrm{W}}_{\mathrm{mn}}^{-1}=\mathrm{w}_{\mathrm{N}}^{-\mathrm{mn}} / \mathrm{N}, \quad \mathrm{w}_{\mathrm{N}}=\exp (-\mathrm{i} 2 \pi / \mathrm{N})
$$

where for clarity we used the arrow and the triangular hat over-scripts to designate vectors and matrices respectively; also, the dot signifies dot product or matrix multiplication. To make possible the matrix multiplication we assume that the vectors are columns, matrices with $N$ rows and 1 column, a practice we will continue throughout the subsection. Actually the convention is that in any indexed expression the first index represents the row and the second the column. The absence of the second index indicates we deal with a column or a vector. More than three indexes means we deal with a tensor and this cannot be intuitively represented easily. Of course the values $G_{n}$ do not equal the corresponding samples of the continuous Fourier transform, but they approximate them. The greater the $N$, the better the approximation will be.

The 2D discrete Fourier transform may be obtained easily by generalizing Eqs. (30-32). Namely, 2D DFT has the form

$$
G_{p q}=\sum_{m=-M / 2}^{M / 2-1} \sum_{n=-N / 2}^{N / 2-1} g_{m n} \exp \left[-i 2 \pi\left(\frac{p m}{M}+\frac{q n}{N}\right)\right]=\sum_{m=-M / 2}^{M / 2-1} \sum_{n=-N / 2}^{N / 2-1} g_{m n} w_{M}^{p m} w_{N}^{q n}
$$

where $M \times N$ is the dimension of the matrix of samples $g_{m n}$ and, consequently, the dimension of the matrix of the Fourier coefficients, or of the DFT $G_{p q}$, with $M$ and $N$ completely unrelated, and we also have the short hand notations

$$
\mathrm{w}_{\mathrm{M}}=\exp (-\mathrm{i} 2 \pi / \mathrm{M}), \mathrm{w}_{\mathrm{N}}=\exp (-\mathrm{i} 2 \pi / \mathrm{N})
$$

The inverse discrete Fourier transform has, of course, the form

$$
\mathrm{g}_{\mathrm{mn}}=\frac{1}{\mathrm{MN}} \sum_{\mathrm{p}=-\mathrm{M} / 2}^{\mathrm{M} / 2-1} \sum_{\mathrm{q}=-\mathrm{N} / 2}^{\mathrm{N} / 2-1} \mathrm{G}_{\mathrm{pq}} \exp \left[\mathrm{i} 2 \pi\left(\frac{\mathrm{pm}}{\mathrm{M}}+\frac{\mathrm{qn}}{\mathrm{N}}\right)\right]=\frac{1}{\mathrm{MN}} \sum_{\mathrm{p}=-\mathrm{M} / 2 \mathrm{q}=-\mathrm{N} / 2}^{\mathrm{M} / 2-1} \mathrm{~g}_{\mathrm{mn}}^{\mathrm{N} / 2-1} \mathrm{w}_{\mathrm{M}}^{-\mathrm{pm}} \mathrm{w}_{\mathrm{N}}^{-\mathrm{qn}}
$$

The linearity of the Fourier transform in Eq. (32) permits the matrix formulation of the direct and inverse 1D DFT. However the generalization to the 2D DFT leads us to a multidimensional matrix formulation:

$$
\hat{\mathrm{G}}_{(2)}=\hat{\mathrm{W}}_{(4)} \cdot \hat{\mathrm{g}}_{(2)}, \quad \hat{\mathrm{g}}_{(2)}=\hat{\mathrm{W}}_{(4)}^{-1} \cdot \hat{\mathrm{G}}_{(2)}, \quad \hat{\mathrm{W}}_{\mathrm{pqmn}}=\mathrm{w}_{\mathrm{N}}^{\mathrm{pm}} / \mathrm{Mw}^{\mathrm{qn}} / \mathrm{N}, \quad \hat{\mathrm{W}}_{\mathrm{mnpq}}^{-1}=\mathrm{w}_{\mathrm{M}}^{-\mathrm{mp}} \mathrm{W}_{\mathrm{N}}^{-\mathrm{nq}}
$$

where $\hat{\mathrm{g}}_{(2)}$ and $\hat{\mathrm{G}}_{(2)}$ are tensors of rank 2 (ordinary $2 \mathrm{D}$ matrices) and $\hat{\mathrm{W}}_{(4)}$ and $\hat{\mathrm{W}}_{(4)}^{-1}$ are tensors of rank 4 . The direct and the inverse Fourier transforms are dot products of the tensors $\hat{\mathrm{W}}_{(4)}$ and $\hat{\mathrm{W}}_{(4)}^{-1}$ with the 2D matrices $\hat{\mathrm{g}}_{(2)}$ and $\hat{\mathrm{G}}_{(2)}$. The dot product of two tensors results in tensors with the rank equal to the sum of the tensors rank minus 2. Eqs. (33-36) are actually those with which one deals when operating 2D discrete Fourier transforms and not Eqs. (29-32). Eqs. (33-36) may seem complicated but the mastery of Eqs. (29-32) leads easily to the multidimensional forms. The term "tensor" was introduced for the sake of completeness but it does not change the simple elementary aspect of Eqs. (29-32) that are 
expressed in tensor form in Eq. (36). For instance one may notice that the 2D DFT is actually two 1D DFTs applied first to the rows of the input matrix then to the resulted columns, although the order of the operations does not matter because the end result is the same.

The direct computation of all the samples $G_{n}$ requires an amount of computation proportional to $N^{2}$. However, the $\hat{W}$ matrix has some special properties that enable massive reduction of the operations required to perform the matrix multiplication $\hat{W} \cdot \vec{g}$. As far back as 1965 a method to compute the discrete Fourier transform by a very much reduced number of operations, the FFT algorithm, which allows computing the discrete Fourier transform with a very high efficiency is known. Originally designed for samples with the number of elements $N$ being powers of 2, now FFT may be calculated for samples with any number of elements, even, what is quite astonishing, non-integer N. A fast algorithm for computing a generalized version of the Fourier transform named the scaled or fractional Fourier transform was also designed. The normalization factors from (29-32) of the direct and the inverse transforms are a matter of convention and convenience, but they must be carefully observed for accurate calculations once a convention was chosen.

Since subroutines for FFT calculations are widely available, there is no need to discuss here in detail the FFT formalism. For the interested reader we recommend (Press et al., 2002), chapter 12 . We will only mention that the algorithm makes use of the symmetry properties of the matrix multiplication by the techniques called time (or space) decimation and frequency decimation, techniques that can be applied multiple times to the input in its original and the intermediary states, and with each application the computation time is almost halved. The knowledge of the FFT algorithm in detail may help the programmer also with the memory management, if that is a problem, because it shows one how to break the input data into smaller blocks, performs FFT separately for each of them and reunites them at the end.

\section{Conversion of the input data for use by the FFT and conversion of the data generated by the FFT in order to have physical meaning}

\subsection{The transposition method}

As we said, one does not need to know in detail the FFT algorithm in order to use it. The FFT subroutines can be used to a large extent as simple black boxes. There is, however, one fact about FFT that even the layman needs to know it in order to use the FFT subroutines. Namely, for mathematical convenience the DFT is not expressed in a physical manner as in Eqs. (29-32) where the current index runs not from $-N / 2$ to $N / 2-1$ but from 0 to $N-1$ :

$$
\begin{gathered}
\mathrm{G}_{\mathrm{n}}=\sum_{\mathrm{m}=0}^{\mathrm{N}-1} \mathrm{~g}_{\mathrm{m}} \mathrm{w}^{\mathrm{mn}}, \mathrm{n}=0,1, \ldots, \mathrm{N}-1 \\
\mathrm{~g}_{\mathrm{m}}=\frac{1}{\mathrm{~N}} \sum_{\mathrm{n}=0}^{\mathrm{N}-1} \mathrm{G}_{\mathrm{n}} \mathrm{w}^{-\mathrm{mn}}, \mathrm{m}=0,1, \ldots, \mathrm{N}-1
\end{gathered}
$$

This shifting of the index allows the application of the decimation techniques we talked about, but also has the effect of a transposition of the wings of the input and as a consequence the, say, "mathematical" output is different than the "physical" output, the one that resembles what one obtains in a practical experiment, although the two outputs are, of 
course, closely connected. The reference (Logofătu \& Apostol, 2007) shows that in order for formulae $(37,38)$ to work the wings of the input vector should be transposed before the application of the FFT procedure and then the wings of the output vector should be transposed back all in a manner consistent with the parity of the number of samples. Namely for even $N$ the input and the output vectors are divided in equal wings. However, for odd $N$ the right wing of the input starts with the median element, therefore is longer with one element; but in the case of the output it is the left wing which contains the median element and is longer. This transposition of the wings is the same thing as the rotation of the elements with $N / 2$ when $N$ is even, and $(N-1) / 2$ when $N$ is odd. For the case of odd $N$ the direction of the rotation is left for the input and right for the output. For even $N$ the direction does not matter.

In a 2D case when both $M$ and $N$ are even the phase change due to the "mathematical" transpositions is just an alternation of signs, in a chess board style. In other situations the phase change is more complicated. It is true that in most cases it is the amplitude spectrum that matters most, but sometimes the phase cannot be neglected and the transposition or rotation operations mentioned above have to be performed. In the 2D case the transpositions do not have to be a double series of wing transpositions for rows and columns. One can make just two diagonal transpositions of the quadrants of the input and output matrices. The division of the input and output matrices depends on the parity of $M$ and $N$. For even $M$ and $N$ things are simple again. The matrices are divided in four equal quadrants. When one of the dimensions is odd the things get complicated, but here again we have a simple rule of thumb. If the number of rows $M$ is odd, then the left quadrants of the input matrix have the larger number of rows (one more) while the left quadrants of the output matrix have the smaller number (one less). For odd $N$ the lower quadrants of the input matrix have the larger number of columns (one more) while the lower quadrants of the output matrix have the smaller number (one less). And viceversa.

\subsection{The sign method}

In addition to the procedure with the transposition of the input before the FFT and the inverse transposition of the output after the FFT, there is another solution for reconciling the results of the mathematical calculation with the physics. It can be done by substituting the indexes of DFT with the indexes used by FFT, thus expressing DFT in the terms preferred by FFT. The expression of DFT that we use is the one from Eq. (31)

$$
G_{n}=\sum_{m=-N / 2}^{N / 2-1} g_{m} \exp \left(-i 2 \pi \frac{m n}{N}\right)
$$

We need to make a reindexation of (39) so that it looks like (37). Let the integer indexes $p$ and $q$ be so that

$$
\begin{aligned}
& \mathrm{p}=\mathrm{m}+\mathrm{N} / 2 \text { or } \mathrm{m}=\mathrm{p}-\mathrm{N} / 2, \mathrm{p}=0,1, \ldots, \mathrm{N}-1 \\
& \mathrm{q}=\mathrm{n}+\mathrm{N} / 2 \text { or } \mathrm{n}=\mathrm{q}-\mathrm{N} / 2, \mathrm{q}=0,1, \ldots, \mathrm{N}-1
\end{aligned}
$$

which means we can introduce two similar sets of samples $g^{\prime}$ and $G^{\prime}$, having the indexes shifted by $N / 2$

$$
\mathrm{g}_{\mathrm{p}}^{\prime}=\mathrm{g}_{\mathrm{p}-\mathrm{N} / 2}=\mathrm{g}_{\mathrm{m}}, \mathrm{G}_{\mathrm{q}}^{\prime}=\mathrm{G}_{\mathrm{q}-\mathrm{N} / 2}=\mathrm{G}_{\mathrm{n}}
$$


Introducing (40) and (41) in (39) we obtain

$$
\mathrm{G}_{\mathrm{n}}=\mathrm{G}_{\mathrm{q}}^{\prime}=\sum_{\mathrm{p}=0}^{\mathrm{N}-1} \mathrm{~g}_{\mathrm{p}}^{\prime} \exp \left[-\mathrm{i} 2 \pi \frac{(\mathrm{p}-\mathrm{N} / 2)(\mathrm{q}-\mathrm{N} / 2)}{\mathrm{N}}\right]=(-1)^{\mathrm{N} / 2}(-1)^{\mathrm{q}} \sum_{\mathrm{p}=0}^{\mathrm{N}-1}\left[(-1)^{\mathrm{p}} \mathrm{g}_{\mathrm{p}}^{\prime}\right] \exp \left(-\mathrm{i} 2 \pi \frac{\mathrm{pq}}{\mathrm{N}}\right)
$$

Therefore, in order to obtain physically meaningful results all we need to do is to multiply the input data with an array consisting in alternating signs and starting with +1 , perform the FFT and then multiply the result again with the same sign array and an overall $(-1)^{N}$ sign. Then we can identify the $G_{q}^{\prime}$ element of the final output with $G_{n}$, the desired element.

For odd $N$ the sign method cannot be applied as such. Instead of signs we have exponentials with imaginary arguments. Although it has a more messy appearance the conversion method is still simple for odd $N$ too. The new indexes are

$$
\begin{aligned}
& \mathrm{p}=\mathrm{m}+(\mathrm{N}-1) / 2 \text { or } \mathrm{m}=\mathrm{p}-(\mathrm{N}-1) / 2, \mathrm{p}=0,1, \ldots, \mathrm{N}-1 \\
& \mathrm{q}=\mathrm{n}+(\mathrm{N}-1) / 2 \text { or } \mathrm{n}=\mathrm{q}-(\mathrm{N}-1) / 2, \mathrm{q}=0,1, \ldots, \mathrm{N}-1
\end{aligned}
$$

The conversion formula is

$$
\mathrm{G}_{\mathrm{n}}=\mathrm{G}_{\mathrm{q}}^{\prime}=\exp \left[-\mathrm{i} \pi \frac{(\mathrm{N}-1)^{2}}{2 \mathrm{~N}}\right] \exp \left(\mathrm{i} \pi \mathrm{q} \frac{\mathrm{N}-1}{\mathrm{~N}}\right) \sum_{\mathrm{p}=0}^{\mathrm{N}-1}\left[\exp \left(\mathrm{i} \pi \mathrm{p} \frac{\mathrm{N}-1}{\mathrm{~N}}\right) \mathrm{g}_{\mathrm{p}}^{\prime}\right] \exp \left(-\mathrm{i} 2 \pi \frac{\mathrm{pq}}{\mathrm{N}}\right)
$$

Instead of sign array one has to use an array with the complex unitary elements $\exp [\mathrm{i} \pi p(\mathrm{~N}-$ 1)/N] with $p$ running from 0 to $N-1$. One has to multiply the input data with this array before feeding it to the FFT procedure. The outcome must be multiplied again with this array and with an overall constant factor $\exp \left[-\mathrm{i} \pi(N-1)^{2} /(2 N)\right]$. The generalization to $2 \mathrm{D}$ is straightforward in both cases. This method, although somewhat similar to the one shown in (Logofătu \& Apostol, 2007) is actually better and simpler.

\section{Correspondences to reality}

A particular case in which we may talk in a sense of "naturally" sampled input functions is the case of binary masks (transmittance 0 or 1 ) that are formed out of identical squares. A 1D grating as in Fig. 1.a (mask A), or a 2D grating as in Fig. 1.b (mask B) are such examples. We chose those masks because our purpose is to compare the discrete and the continuous Fourier transforms and for those masks CFT can be calculated analytically. The masks are not sampled functions in the sense of Eq. (9), there are no delta functions in their expression. They are, however, sampled in the sense that for evenly spaced rectangular areas the transparency functions are constant, hence, the functions can be represented, as discrete matrices of samples. We chose masks with a low number of samples, only $32 \times 32$, not because DFT is difficult or time-consuming (actually, due to the existence of the FFT algorithm DFT can be done very quickly for quite a large number of samples), but to make easier the calculation of CFT, which is indeed considerably time-consuming. Also, at small number of samples the differences between the discrete and the continuous spectrum can be more easily seen. The period of the gratings, both horizontally and vertically, was chosen to be 7 squares, so that it does not divide 32; in this way the Fourier spectrum gets a little complicated and we avoid symmetry effects that may obscure the points we want to make. 


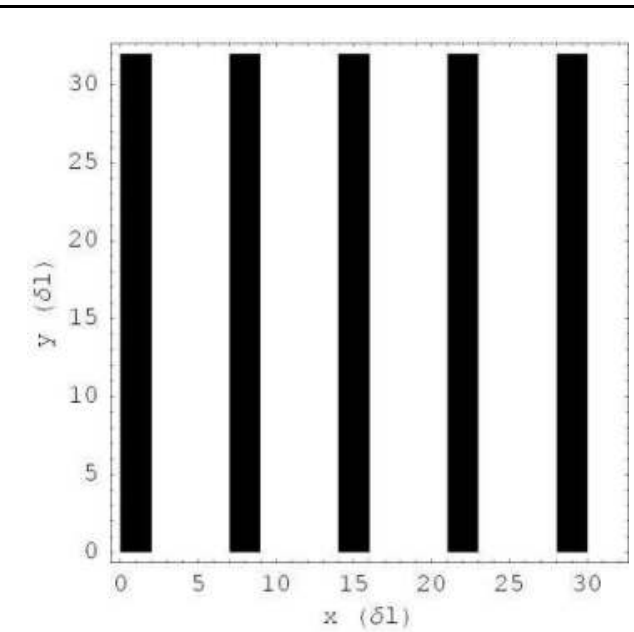

(a)

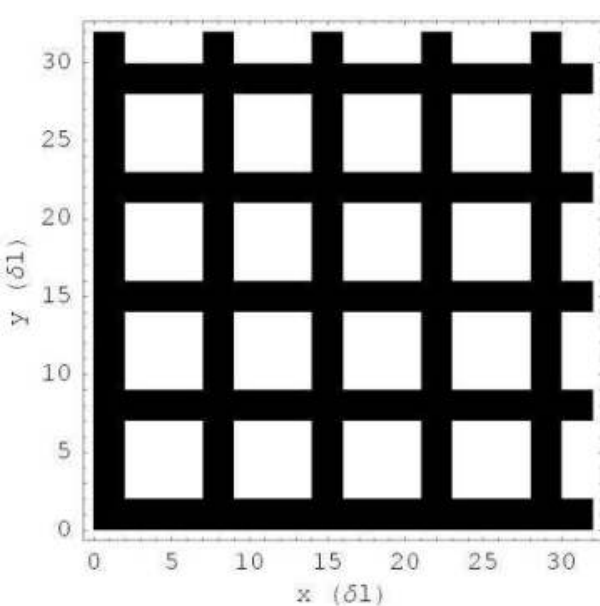

(b)

Fig. 1. Binary masks: a) $1 D$ grating and b) $2 D$ grating. Both gratings consist in $32 \times 32$ squares, black or white, of equal dimension $\delta l$. We labelled the two masks " $\mathrm{A}$ " and "B". It is assumed that outside the represented area of the masks the input light is completely blocked, i.e. the transparency function is zero.

It seems natural to represent the masks from Fig. 1, for DFT calculations, as $32 \times 32$ matrices with values 1 or 0 corresponding to the transmission coefficient of the squares of the masks. The squared absolute values of the DFT of the previously discussed type of matrix for mask $\mathrm{A}$ and the CFT of mask A are shown together in Fig. 2. The square absolute value is the power or the luminous intensity, the only directly measurable parameter of the light field. The CFT of mask A has the expression

$$
G\left(f_{x}, f_{y}\right)=\delta l \operatorname{sinc}\left(f_{x} \delta l\right) 32 \delta l \operatorname{sinc}\left(32 f_{y} \delta l\right) \sum_{p=1}^{32} g_{p} \exp \left[-i 2 \pi f_{x}(p-1) \delta l\right]
$$

where

$$
\operatorname{sinc}(\mathrm{t}) \equiv \frac{\sin (\pi \mathrm{t})}{\pi \mathrm{t}}
$$

and obviously $N$ is here 32. Since mask A is 1D [in a limited sense only; if it were truly $1 \mathrm{D}$ then the dependence on $f_{y}$ of $G$ would be of the form $\left.\delta\left(f_{y}\right)\right]$ in the calculation of $|G|^{2}$ in Fig. 2 and the following figures that represent $|G|^{2}$ for mask $A$, we discarded the dependence on $f_{y}$ and the entire contribution of the integration over $y$ and we used only the part corresponding to the integration over $x$. The DFT calculations were rescaled (multiplied with $N^{1 / 2}$ ) so that they could be compared to the CFT calculations. All the CFT spectra represented in this article are computer calculations, hence they are simulations. A correctly done experiment of Fourier optics would yield, of course, the same results.

Two types of discrepancies can be noticed in Fig. 2 between CFT and DFT. First they have different values for the same spatial frequency, sometimes there is even a considerable 
difference. Second, the discrete spectrum does not offer sufficient information for the domain of spatial frequencies in between two discrete values, and the interpolation of the sampled values does not lead always to a good approximation. The structure of the continuous spectrum is much richer than that of the discrete spectrum. There are of course many reasons why the discrete Fourier spectrum $\left|G^{s p}\right|^{2}$ is different than the genuine spectrum $|G|^{2}$. One reason is the periodicity. The $g$ input functions are not generally periodic or at least they are not infinitely periodic. The input functions illustrated in Fig. 1 are periodic in the sense they have a limited number of periods, but rigorously periodic means an infinity of periods.

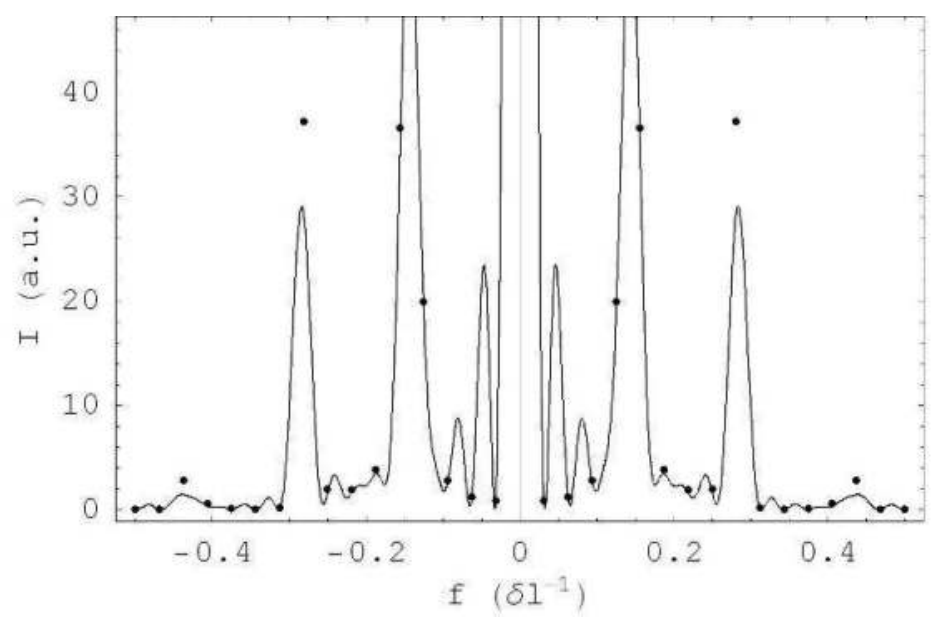

Fig. 2. The continuous (solid line) and the discrete (dots) Fourier power spectra for mask A vs. the spatial frequency shown together. For DFT calculations the "natural" sampling was used. Only the central part of the continuous spectrum for which DFT provides output values is represented. The spatial frequency is expressed in $\delta l^{-1}$ units.

Corresponding to the two types of discrepancies there are also two ways of improving the discrete spectrum. One way is to increase the sampling rate. We can do that by "swelling" the sampling array, inserting more than once the value corresponding to a square. The increased sampling rate improves the agreement between DFT and CFT. We illustrated in Fig. 3 only the calculations for the same spatial frequencies as those represented in Fig. 2, not just to ease the comparison but also because the spectrum outside is very weak and, hence, negligible. One big the difference between the CFT and DFT version represented in Fig. 2 is the $\operatorname{sinc}\left(f_{x} \delta l\right)$ function, that is the Fourier transform of the rectangular function. Because for Fig. 3 by allotting more samples for each square we had a higher sampling rate, the rectangular shape was felt in the DFT calculations. We increased the sampling rate 10 times and, as one can see in Fig. 3, the continuous and the discrete spectrum are now closer, they are almost on top of each other. Because there are now 10 times more elements than in the "natural" sampling, and actually in this new sampling the same elements are just repeated 10 times, in order to be able to compare DFT and CFT we had to divide the DFT spectrum by $10^{2}$. 


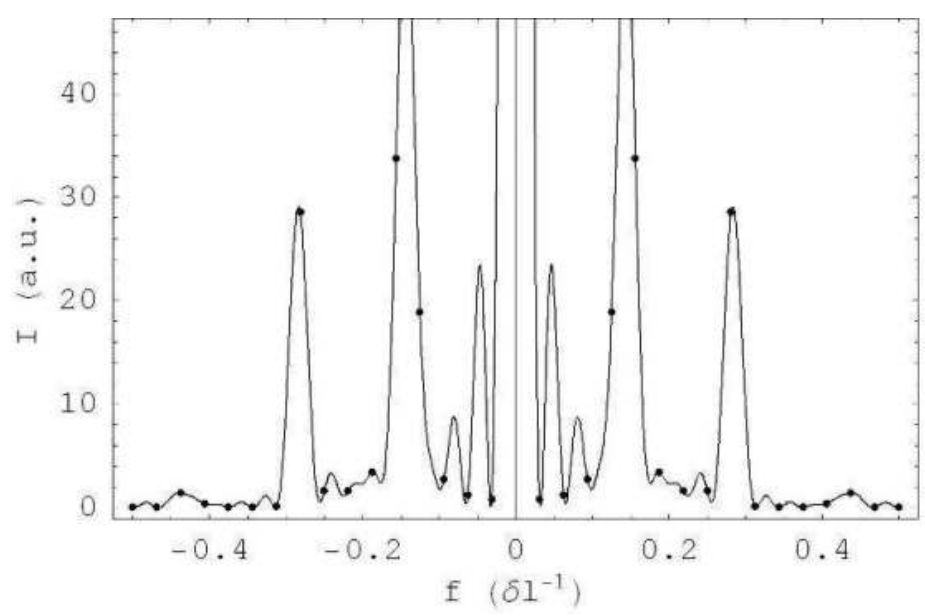

Fig. 3. The continuous (solid line) and the discrete (dots) Fourier power spectra for mask A vs. the spatial frequency shown together. For the DFT calculations was used a higher (10 times) sampling rate of mask A than that used for the DFT calculations shown in Fig. 2. Only the portion of the both spectra corresponding to the spatial frequency range of Fig. 2 is shown.

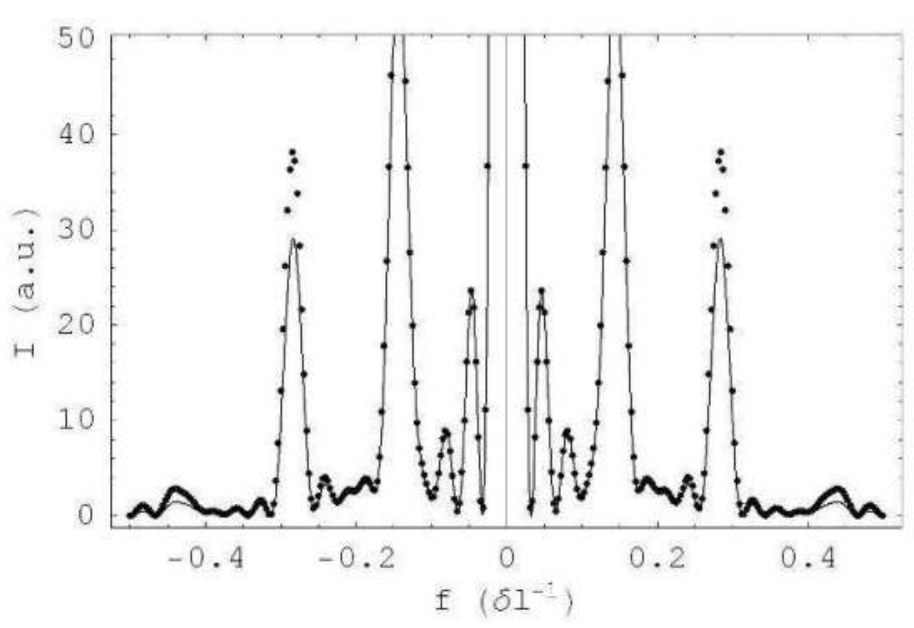

Fig. 4. The continuous (solid line) and the discrete (dots) Fourier power spectra for mask A vs. the spatial frequency shown together. The sampling of mask A was extended so that to include part of the surrounding darkness shown together, maintaining the same sampling rate as the "natural" sampling. Compared to the original sampling used for the calculations of Fig. 2, the sampling was now extended 10 times, which accounts for the higher density of dots of the DFT output, which now numbers 320 samples. Although in the figure 320 points are represented, the spatial frequency range is the same as that of Figs. 2 and 3. 
Another way to improve the conformity of the DFT to CFT is to increase the sampled area. Since outside the area of mask A there is no structure, only darkness, and $g$ is just zero, when making the sampling of $g$ we are generally tempted to discard this surrounding darkness. But when we express DFT in terms of CFT, as we saw in section 3.2, we consider that the structure of mask A is periodically repeated back to back in what we know to be just darkness. Therefore, in order to improve the similarity of DFT to reality (which is CFT), it is a good idea to pad the original sampled function with zeros to the left and to the right to account for the surrounding darkness. We padded with zeros so that the original sampled array of values was increased 10 times. In Fig. 4 the squared absolute values of the DFT and CFT for the new sampled function are again compared, and, although their values are still different, now DFT offers more information, enough for interpolation. We did not need here another type of rescale of DFT than that done in Fig. 2 in order to have a meaningful comparison to CFT, because the new elements added to the input sampling were just zeros.

The two procedures for improving the similarity of DFT to CFT described above and illustrated in Figs. 3 and 4 may be combined and the result is shown in Fig. 5. The sampling array used for the calculation of DFT is now both "swollen" and extended, having 100 times more elements than the "natural" sampling. Now DFT is both closer to CFT and richer in information. Now DFT is both accurate and able to provide enough information for a correct interpolation.

It should be noted that in Figs. 2-5 only the discrete spectrum changes, the continuous spectrum is a constant reference.

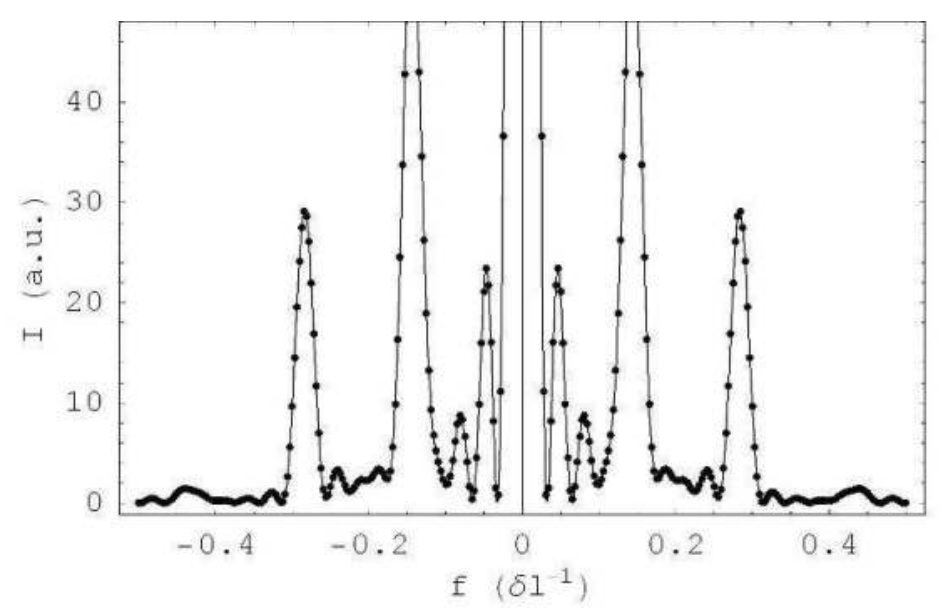

Fig. 5. The continuous (solid line) and the discrete (dots) Fourier power spectra for mask A vs. the spatial frequency shown together. For DFT calculations the sampling was both extended and its rate increased. An array of $32 \times 10 \times 10$ points was used, but only the $32 \times 10$ points corresponding to the spatial frequency range of Figs. 2-4 were shown.

The similar procedure applied to mask A was also applied to mask B. We found appropriate to illustrate the procedure for mask $B$ because optics is generally about images and these are 2D, not 1D, which is just a particular case, useful mostly for the easiness of the graphic representation than for practical purposes. The Fourier spectrum of a $2 \mathrm{D}$ mask is more 
difficult to represent. We chose to represent the spectrum as levels of grey. Moreover, to simulate the vision of the eye we represented the logarithm of the luminous intensity. Another reason for using the logarithmic representation is the fact that the Fourier spectrum decreases quite sharply with the spatial frequency and only the representation of the logarithm allows the fine shades to be visible.

In Fig. 6 the represented continuous Fourier spectrum is the logarithm of the squared absolute value of the function

$$
G\left(f_{x}, f_{y}\right)=\delta l^{2} \operatorname{sinc}\left(f_{x} \delta l\right) \operatorname{sinc}\left(f_{y} \delta l\right) \sum_{p=1}^{32} \sum_{q=1}^{32} g_{p q} \exp \left[-i 2 \pi\left(f_{x}(p-1)+f_{y}(q-1)\right) \delta l\right]
$$

In Fig. 7 it is represented the discrete Fourier spectrum of the "natural" sampling of mask B, that is of a $32 \times 32$ matrix with each element having the value of the corresponding square element of mask B, 0 or 1 . The comparison of Figs. 6 and 7 shows marked differences. Just as in the case of mask A we tried next to compensate for the shortcomings of the "natural" sampling by extending the sampling and increasing the sampling rate. The result is right on top of Fig. 6, so we did not consider necessary to represent it graphically.

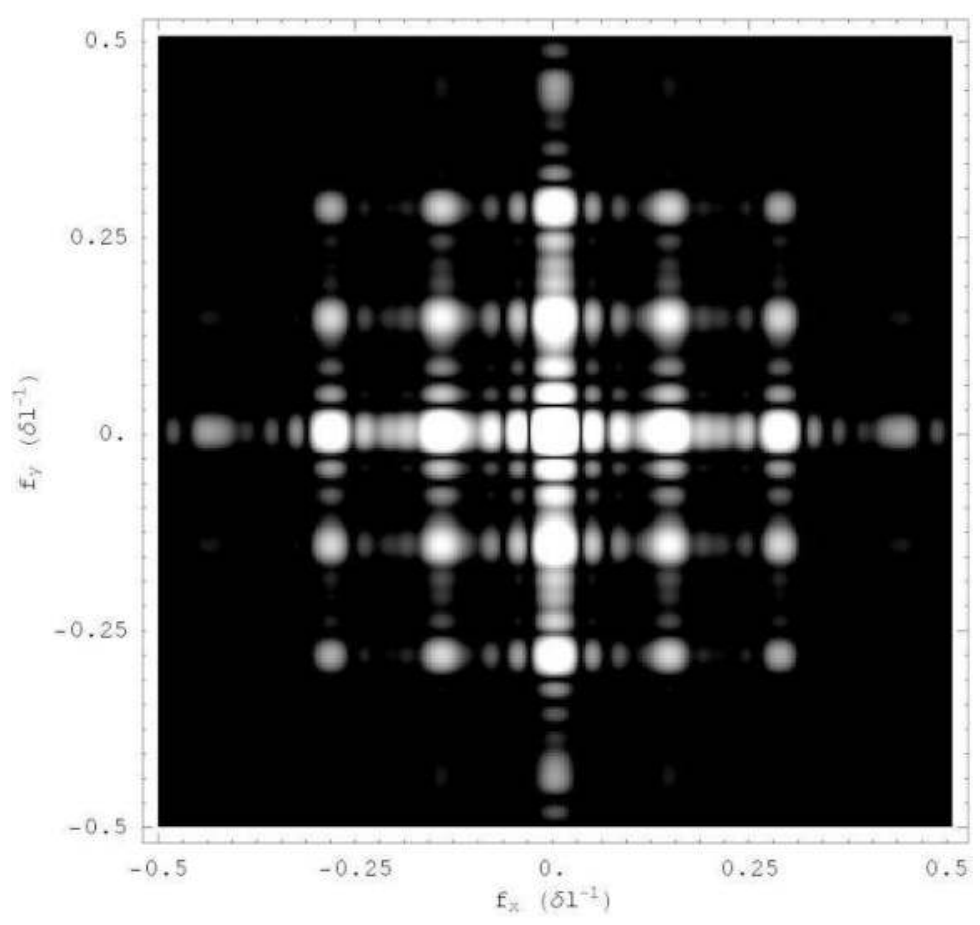

Fig. 6. The central high intensity portion of the continuous Fourier spectrum of mask B, chosen so that to match the spatial frequency ranges of the DFT of the "naturally" sampled input of mask B (see Fig. 7 below). The abscissa and the ordinate are the spatial frequencies and the light intensity of the spectrum is coded as levels of grey. 


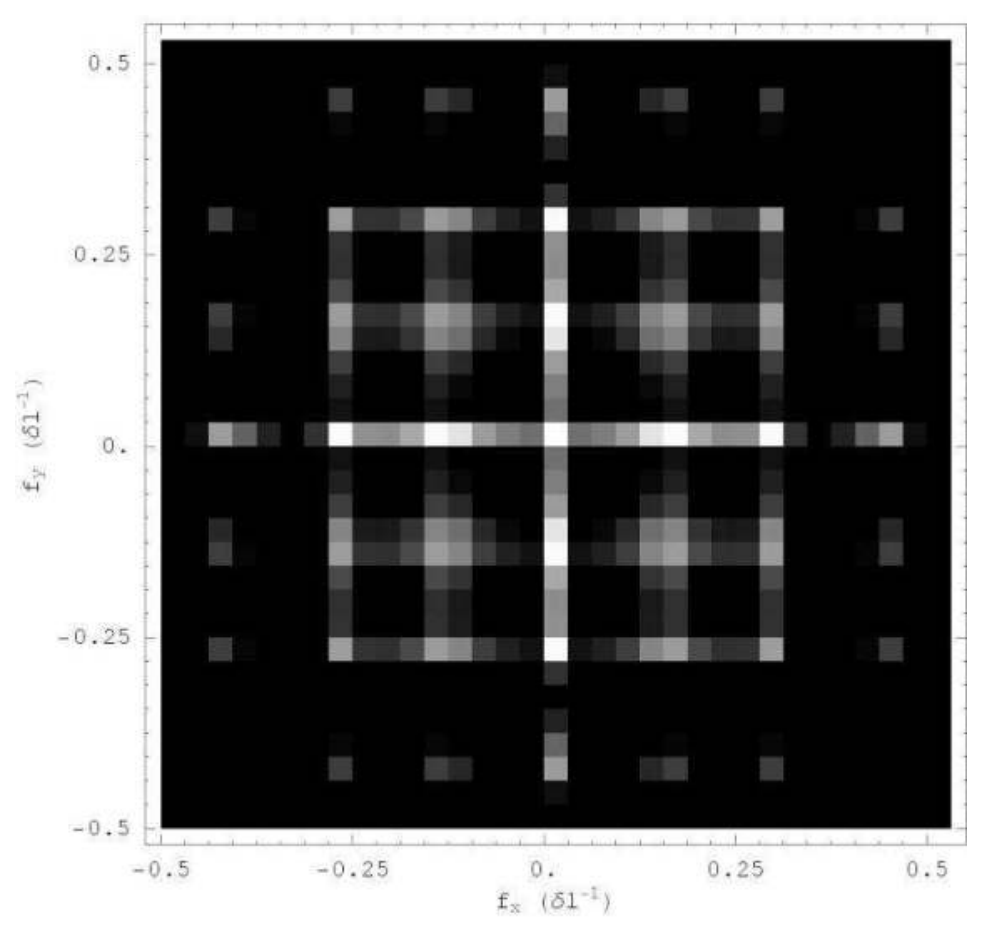

Fig. 7. The discrete Fourier spectrum of the "natural" sampling of mask B. The abscissa and the ordinate are the spatial frequencies, and the light intensity is codes as levels of grey, just as in Fig. 6.

As a side comment, one may notice that all the spectra shown in this article are symmetric. The 1D plots are symmetric with respect to the origin, and the 2D plots are symmetric with respect to the vertical axis. There is a redundancy of information. The 1D plots contain in one horizontal half all the information, and the 2D plots contain all the information in any of the 4 quadrants. This is due to the fact that we calculated the spectra of transmission masks that do not modify the phase of the input optical fields and we assumed the input wave to be a plane wave, which is actually a common situation in Fourier analysis. It is this property of the masks that causes the spectra to be symmetric. Rigorously speaking they are not symmetric, the phase differ in the two halves of the 1D plots and in the 2D plots the phase of two diagonal quadrants differs from that of the other two diagonal quadrants. But the difference is just that they have conjugate complex values. The absolute values of two conjugate complex quantities is the same, hence the 4 -fold symmetry of the $2 \mathrm{D}$ power spectra.

To give some dimensional perspective to the considerations presented in this subsection, it might be instructive to give a value to $\delta l$ and to specify the experimental conditions in which the Fourier transform is performed. We need very small masks in order to make the Fourier spectrum macroscopic, but also large enough so that sufficient light passes through and the Fourier spectrum is visible. $100 \mu \mathrm{m}$ is such a value for $\delta l$. Then the masks A and B would be 
squares of $3.2 \mathrm{~mm}$ dimension. The Fourier spectra (continuous or discrete) represented in Figs. 2-8 are segments for the 1D case and squares for the $2 \mathrm{D}$ case having the dimension $\Delta f=1 / \delta l=10 \mathrm{~mm}^{-1}$ in spatial frequency units. If the Fourier transform is performed by a lens of focal length $F=1 \mathrm{~m}$ and the light source is a He-Ne laser of wavelength $\lambda=632.8 \mathrm{~nm}$, then dimensions of both spectra in the Fourier plane (back focal plane of the lens) are identical $\lambda F \Delta f=6.328 \mathrm{~mm}$.

\section{Conclusion}

The problem of the relation between DFT and CFT is investigated in this article. In order to understand the physical meaning of DFT we expressed it in terms of CFT. The Fourier series was a useful tool in this endeavour, because it is an intermediary link between CFT and DFT. Namely, the two properties of both the input function and the Fourier spectrum of DFT, periodicity and discrete character, are present in the Fourier series, except that the input function is just periodic and the Fourier spectrum is just discrete. The connection between periodicity and the discrete character is stressed, namely it is shown that the periodicity of the input/output implies a discrete character of the output/input and viceversa. For convenience the derivations were made for 1D input functions but they can be easily and straightforwardly extended to 2D input functions, if the sampling is done over two mutually perpendicular directions and the sampled area is rectangular. It is shown that DFT is the CFT of a periodic input of delta functions in which case the output is also periodic and composed of delta functions. The incongruence between DFT and CFT indicates that DFT may not be a good approximation of CFT, and some numerical examples prove it. Two masks, first $1 \mathrm{D}$ and the second $2 \mathrm{D}$ were studied with respect to the agreement of their discrete with their continuous Fourier spectra. It has been shown that if the sampling rate and the extension of the masks are properly chosen (large enough) DFT is a good approximation of CFT. No generally valid criterion for the agreement between DFT and CFT is given, only the ways of improving it are indicated and shown to be sufficient for the particular cases studied in this article.

Our previous attempts to bridge the gap between CFT and DFT, between physics and mathematics (Logofătu and Apostol, 2007; Nascov et al, 2010) were by no means a closed and shut subject but rather were intended as an opening of new avenues of research. Some details, usually left out by other authors, such as the transposition of the input data done for the application of the FFT algorithm are explained and two solutions for dealing with the problem are presented. The second solution, presented in subsection 4.2 even shows how the transposition of the input leaving the amplitude unchanged modifies the phase with a linear progressive phase function.

\section{References}

Apostol, D.; Sima, A.; Logofătu, P. C.; Garoi, F.; Damian, V., Nascov, V. \& Iordache, I. (2007). Static Fourier transform lambdameter, Proceedings of ROMOPTO 2006: Eighth Conference on Optics, Vol. 6785, pp. 678521, ISSN 0277-786X, Sibiu, Romania, August 2007, SPIE, Bellingham, WA (a)

Apostol, D.; Sima, A.; Logofătu, P. C.; Garoi, F.; Nascov, V.; Damian, V. \& Iordache, I. (2007). Fourier transform digital holography, Proceedings of ROMOPTO 2006: Eighth 
Conference on Optics, Vol. 6785, pp. 678522, ISSN 0277-786X, Sibiu, Romania, August 2007, SPIE, Bellingham, WA (b)

Arfken, G. B. \& Weber H. J. (2001). Mathematical methods for physicists, Harcourt / Academic Press, ISBN 0-12-059826-4, San Diego, chapters 14,15

Bailey, D. H. \& Swarztrauber, P. N. (1991). The Fractional Fourier Transform and Applications, SIAM Review, Vol. 33, No. 3, pp. 389-404, ISSN 0036-1445

Bracewell, R. (1965) The Fourier Transform and Its Applications, McGraw-Hill, New York

Brigham, E. O. (1973) The Fast Fourier Transform: An Introduction to Its Theory and Application, Prentice-Hall, Englewood Cliffs NJ

Bringdahl, O. \& Wyrowski, F. 1990. Digital holography - computer generated holograms, In Progress in Optics vol, 28, E. Wolf, (Ed.), pp. 1-86, Elsevier B.V. ISBN: 9780444884398

Collier, R. J.; Burckhardt, C. B. \& Lin, L. H. (1971) Optical holography, Academic Press, New York

Cooley, J. W. \& Tukey, J. W. (1965). An algorithm for the machine computation of the complex Fourier series, Mathematics of Computation, Vol. 19, No. 90, pp. 297-301, ISSN 0025-5718

Goodman, J. W. (1996). Introduction to Fourier Optics, McGraw Hill, ISBN 0-07-024254-2, New York

Lee, W.-H. (1978). Computer-Generated Holograms: Techniques and Applications, In Progress in Optics vol. 16, E. Wolf, (Ed.), pp. 119-232, Elsevier B. V., ISSN 00796638,

Lohmann, A. W. \& Paris, D. P. (1967). Binary Fraunhofer holograms, generated by computer, Applied Optics Vol. 6, No. 10, pp. 1739-1748, ISSN 0003-6935

Logofătu, P. C. \& Apostol, D. (2007). The Fourier transform in optics: from continuous to discrete or from analogous experiment to digital calculus, Journal of Optoelectronics and Advanced Materials, Vol. 9, No. 9, pp. 2838-2846, ISSN 1454-4164

Logofătu, P. C.; Sima, A. \& Apostol, D. (2009). Diffraction experiments with the spatial light modulator: the boundary between physical and digital optics, Proceedings of Advanced Topics in Optoelectronics, Microelectronics, and Nanotechnologies IV Vol. 7297, pp. 729704, ISSN 0277-786X, Constanta, Romania, January 2009, SPIE, Bellingham WA

Logofătu, P. C.; Garoi, F.; Sima, A.; Ionita, B. \& Apostol, D. (2010). Classical holography experiments in digital terms, Journal of Optoelectronics and Advanced Materials, Vol. 12 No. 1, pp. 85-93, 1454-4164

Nascov, V. \& Logofătu, P. C. (2009). Fast computation algorithm for the RayleighSommerfeld diffraction formula using a type of scaled convolution, Applied Optics Vol. 48, No. 22, pp. 4310-4319, ISSN 0003-6935

Nascov, V.; Logofătu, P. C. \& Apostol, D. (2010). The Fourier transform in optics: from continuous to discrete (II), Journal of Optoelectronics and Advanced Materials, Vol. 12, No. 6, pp. 1311-1321, ISSN 1454-4164

Press, W. H.; Teukolsky, S. A.; Vetterling, W. T. \& Flannery, B. P. (2002). Numerical Recipes in C++, Cambridge University Press, ISBN 0-571-75033-4, Cambridge

Yaroslavsky, L. \& Eden, M. (1996) Fundamentals of digital optics, Birkhäuser, ISBN 0-81763822-9, Boston 


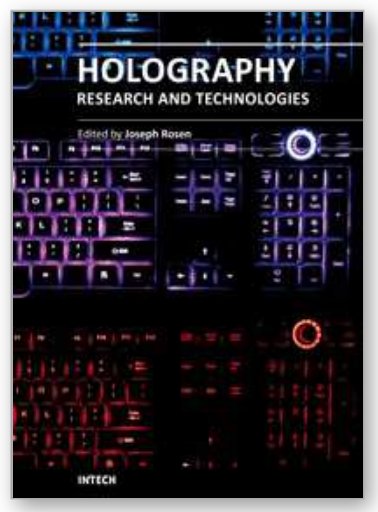

\author{
Holography, Research and Technologies \\ Edited by Prof. Joseph Rosen
}

ISBN 978-953-307-227-2

Hard cover, 454 pages

Publisher InTech

Published online 28, February, 2011

Published in print edition February, 2011

Holography has recently become a field of much interest because of the many new applications implemented by various holographic techniques. This book is a collection of 22 excellent chapters written by various experts, and it covers various aspects of holography. The chapters of the book are organized in six sections, starting with theory, continuing with materials, techniques, applications as well as digital algorithms, and finally ending with non-optical holograms. The book contains recent outputs from researches belonging to different research groups worldwide, providing a rich diversity of approaches to the topic of holography.

\title{
How to reference
}

In order to correctly reference this scholarly work, feel free to copy and paste the following:

Petre Cătălin Logofătu, Victor Nascov and Dan Apostol (2011). The Fourier Transform in Optics: Analogous Experiment and Digital Calculus, Holography, Research and Technologies, Prof. Joseph Rosen (Ed.), ISBN: 978-953-307-227-2, InTech, Available from: http://www.intechopen.com/books/holography-research-andtechnologies/the-fourier-transform-in-optics-analogous-experiment-and-digital-calculus

\section{INTECH}

open science | open minds

\section{InTech Europe}

University Campus STeP Ri

Slavka Krautzeka 83/A

51000 Rijeka, Croatia

Phone: +385 (51) 770447

Fax: +385 (51) 686166

www.intechopen.com

\section{InTech China}

Unit 405, Office Block, Hotel Equatorial Shanghai

No.65, Yan An Road (West), Shanghai, 200040, China

中国上海市延安西路 65 号上海国际贵都大饭店办公楼 405 单元

Phone: +86-21-62489820

Fax: +86-21-62489821 
(C) 2011 The Author(s). Licensee IntechOpen. This chapter is distributed under the terms of the Creative Commons Attribution-NonCommercialShareAlike-3.0 License, which permits use, distribution and reproduction for non-commercial purposes, provided the original is properly cited and derivative works building on this content are distributed under the same license. 